\title{
Same meat, different gravy: ignore the new names of mycobacteria
}

\author{
Enrico Tortoli (10)', Barbara A. Brown-Elliott², James D. Chalmers ${ }^{3}$, \\ Daniela M. Cirillo $\mathbb{0}^{1}$, Charles L. Daley ${ }^{4,5}$, Stefan Emler (1) $^{6}$, R. Andres Floto ${ }^{7}$, \\ Maria J. Garcia ${ }^{8}$, Wouter Hoefsloot $\mathbb{1}^{9}$, Won-Jung Koh ${ }^{10}$, \\ Christoph Lange 11,12,13,14,15, Michael Loebinger ${ }^{16}$, Florian P. Maurer (1) ${ }^{17}$, \\ Kozo Morimoto $^{18}$, Stefan Niemann ${ }^{19,20}$, Elvira Richter ${ }^{21}$, Christine Y. Turenne $^{22}$, \\ Ravikiran Vasireddy ${ }^{2}$, Sruthi Vasireddy ${ }^{2}$, Dirk Wagner (1) ${ }^{23}$, \\ Richard J. Wallace $\mathrm{Jr}^{2}$, Nancy Wengenack ${ }^{24}$ and Jakko van Ingen (1) ${ }^{25}$
}

Affiliations: ${ }^{1}$ Emerging Bacterial Pathogens Unit, IRCCS San Raffaele Scientific Institute, Milan, Italy. ${ }^{2}$ Dept of Microbiology, Mycobacteria/Nocardia Research Laboratory, The University of Texas Health Science Center at Tyler, Tyler, TX, USA. ${ }^{3}$ Scottish Centre for Respiratory Research, University of Dundee, Dundee, UK. ${ }^{4}$ Division of Mycobacterial and Respiratory Infections, National Jewish Health, Denver, CO, USA. ${ }^{5}$ Dept of Medicine, University of Colorado, Aurora, CO, USA. ${ }^{6}$ SmartGene GmbH, Zug, Switzerland. ${ }^{7}$ Cambridge Centre for Lung Infection, Papworth Hospital, Cambridge, UK. ${ }^{8}$ Dept of Preventive Medicine, Public Health and Microbiology, Autonoma University of Madrid, Madrid, Spain. ${ }^{9}$ Radboudumc Center for Infectious Diseases, Dept of Pulmonary Diseases, Radboud University Medical Center, Nijmegen, The Netherlands. ${ }^{10}$ Division of Pulmonary and Critical Care Medicine, Dept of Medicine, Samsung Medical Center, Sungkyunkwan University School of Medicine, Seoul, South Korea. ${ }^{11}$ Clinical Infectious Diseases, Research Center Borstel, Borstel, Germany. ${ }^{12}$ Clinical Tuberculosis Unit, German Center for Infection Research, Borstel, Germany. ${ }^{13}$ Respiratory Medicine and International Health, University of Lübeck, Lübeck, Germany. ${ }^{14}$ Arctic Tuberculosis Center, University of Umeå, Umeå, Sweden. ${ }^{15}$ Dept of Medicine, Karolinska Institute, Stockholm, Sweden. ${ }^{16}$ Host Defence Unit, Royal Brompton Hospital, London, UK. ${ }^{17}$ National Reference Center for Mycobacteria, Research Center Borstel, Borstel, Germany. ${ }^{18}$ Division of Clinical Research, Fukujuji Hospital, Japan Anti-Tuberculosis Association, Tokyo, Japan. ${ }^{19}$ Molecular and Experimental Mycobacteriology, Research Center Borstel, Borstel, Germany. ${ }^{20}$ German Center for Infection Research (DZIF), Partnersite Hamburg-Lübeck-Borstel, Germany. ${ }^{21}$ MVZ Labor Dr. Limbach, Heidelberg, Germany. ${ }^{22}$ Dept of Medical Microbiology and Infectious Diseases, University of Manitoba, Winnipeg, MB, Canada. ${ }^{23}$ Division of Infectious Diseases, Department of Medicine II, University Medical Center Freiburg, Freiburg, Germany. ${ }^{24}$ Division of Clinical Microbiology, Mayo Clinic, Rochester, MN, USA. ${ }^{25}$ Radboudumc Center for Infectious Diseases, Dept of Medical Microbiology, Radboud University Medical Center, Nijmegen, The Netherlands.

Correspondence: Enrico Tortoli, Emerging Bacterial Pathogens Unit, IRCCS San Raffaele Scientific Institute, via Olgettina 60, 20132, Milan, Italy. E-mail: tortoli.enricolahsr.it

@ERSpublications

A new name for most Mycobacterium species has been recently proposed. According to taxonomic rules novel and previous nomenclature coexist and are synonyms. The use of the latter has the advantage of avoiding confusion for healthcare and harm for patients. http://bit.ly/2XhTACc

Cite this article as: Tortoli E, Brown-Elliott BA, Chalmers JD, et al. Same meat, different gravy: ignore the new names of mycobacteria. Eur Respir J 2019; 54: 1900795 [https://doi.org/10.1183/13993003.007952019].

In a recent article, GUPTA et al. [1] have proposed a revision of the taxonomy of mycobacteria with the redistribution of 150 species of the genus Mycobacterium into five different genera; the four newly proposed genera hosting the nontuberculous mycobacteria (NTM) are called Mycobacteroides, Mycolicibacter, Mycolicibacterium and Mycolicibacillus. The proposed taxonomy is based on the theory of synapomorphies, molecular markers that most likely appeared early in an ancestor and then were vertically 
transmitted to descendants [2]. The detection of a number of such molecular markers, represented either by specific amino acid insertions/deletions or by whole proteins, selectively present in evolutionary-related groups of species, clearly depicts five major clades within the conventional genus Mycobacterium. The work is mostly sound and the emerging phylogeny impressively overlaps the ones originated by other phylogenomic approaches [3, 4]. The novelty is that GUPTA et al. [1] have suggested to assign to each of these clades the status of independent genera.

What has caused most confusion among mycobacteriologists was not the study by GUPTA et al. [1], but the validation of the novel nomenclature [5] published by the International Journal of Systematic and Evolutionary Microbiology (IJSEM), the official publication of the International Committee on Systematics of Prokaryotes. We believe the new nomenclature has the potential to cause confusion and provides no benefits to the field of clinical mycobacteriology. Investigating the rules regulating the nomenclature of prokaryotes, unknown to the majority of microbiologists and clinicians, produced surprising results. Accordingly, in this editorial, we aim to provide clinicians involved in the management of patients with NTM disease state of the art information about rules regulating the nomenclature of prokaryotes and that, in spite of this recent publication [1], the currently used nomenclature of NTM can remain unchanged.

The bacterial taxonomy is ruled by the Code of nomenclature of prokaryotes [6] (previously Bacteriological code) that, differently from common assumptions, does not provide instructions on description of novel species nor in making decisions in case of divergent opinions among taxonomists. We were indeed surprised to read that, as stated by Tindall [7], "an 'official' taxonomy has not been introduced" in accordance with the principle of "freedom of taxonomic thought and action".

Actually the Code only provides valid names of bacteria, given that two major conditions are fulfilled: the valid publication and the priority [8]. To be valid, a publication describing a new taxon needs to contain a protologue. The protologue is a short paragraph including the name of the taxon followed by the specification nov. (in case of a new species, sp. nov.; in case of a new genus gen. nov. etc.), the etymology with its syllabication and accentuation, and by a description of the salient features of the new taxon, the designation of the type strain, and the accession numbers certifying its deposition in at least two international culture collections located in different countries. Priority means that in cases where the proposed new name has been previously assigned to another validly published taxon, the latter has the priority and a novel name must be proposed prior to validation.

The publication in IJSEM automatically grants validity to a new name; in contrast, in order for an organism name to be recognised as valid if published in other journals, the authors must request the new name to be included in a Validation list published, upon check of conformity, by IJSEM.

When a change of a validly published species or genus name is proposed, the new name, once validly published becomes a heterotypic synonym of the previous ones. Thus, both are correct (valid) and can be used without restriction. The common thought that the most recent name replaces the previous one is groundless. A validly published name is not ever withdrawn.

The article by GUPTA et al. [1] is indeed validly published (it contains the protologue for all the species taken into account, where the respective type strains are confirmed) and the new names have been included in a validation list by IJSEM [5]. As a consequence, the species allocated in genera other than Mycobacterium (Mycobacteroides, Mycolicibacter, Mycolicibacterium and Mycolicibacillus) have synonyms, both of which can correctly be used. The authors of this editorial, and probably the majority of microbiologists and clinicians, were not acquainted with this possibility.

The nomenclature based on five genera also has two major pitfalls; first, it applies and is validly published with limit to the 150 species investigated by GUPTA et al. [1]; however, at least 40 others continue to have Mycobacterium as the only valid name. Second, four of the genomes investigated in the study of GuPTA et al. [1]: M. rhodesiae, M. szulgai, M. tusciae and M. vulneris were mislabelled [4]. M. rhodesiae and M. tusciae proved to be unidentified rapidly growing mycobacteria; M. szulgai proved to be M. kubicae and M. vulneris proved to be M. porcinum. This means that the transfer of M. vulneris (a slow grower) to the genus Mycolicibacter is incorrect [9].

Overall, the updated nomenclature and its adoption in IJSEM and in databases used for sequence-based strain identification (e.g. GenBank) or MALDI-TOF mass spectra databases does a disservice to microbiologists, clinicians and patients. Nontuberculous mycobacteria may be diverse in their genetics and biology, but they produce remarkably similar disease manifestations in distinct populations at risk. This uniformity and the lessons to be learned from it are important for both clinicians as well as the affected patients. Hence, to repeat the words of Amalio Telenti, now 20 years ago: "clinical meaningfulness should be the key to taxonomic precision" [10]. The pragmatic solution is to choose the nomenclature less prone to errors and, in this view, the one based on the single genus, Mycobacterium, has important advantages. 
Significant progress has been gained in recent years by promoting evidence-based clinical care, treatment outcomes and clinical trials in nontuberculous mycobacterial infections as a group, sharing a simple term familiar to clinicians and patients (mycobacteria) and an appropriate acronym (NTM) [11-14]. The proposed new nomenclature does not help this cause.

As it seems, Gupta et al. [1] did rather not solve a burning taxonomic problem within the genus Mycobacterium; their findings, while being mostly correct and scientifically sound, do however not justify the disruption caused by the splitting and renaming of this medically important genus. Substantial cost and effort will be needed to update procedures and laboratory information management systems in hospitals and laboratories which support physicians and care-givers in their daily work, to enable them to deal with the new names and their synonyms without confusion. All this is in fact without a perceivable advantage for the patients nor healthcare in general.

We are confident that microbiologists and clinicians will clearly support the possibility, offered by the Code, to wilfully ignore renaming of clinically important organisms such as nontuberculous mycobacteria.

Conflict of interest: E. Tortoli has nothing to disclose. B.A. Brown-Elliott has nothing to disclose. J.D. Chalmers has received research grants from GlaxoSmithKline, Boehringer Ingelheim, AstraZeneca, Pfizer, Grifols, Bayer AG, Polyphor and Insmed; and received consultancy, congress travel or speaker fees from GlaxoSmithKline, Bayer Healthcare, Aradigm Corporation, Grifols, Pfizer, Boehringer Ingelheim, Napp and Insmed. D.M. Cirillo has nothing to disclose. C. L. Daley has nothing to disclose. S. Emler has nothing to disclose. R.A. Floto has nothing to disclose. M.J. Garcia has nothing to disclose. W. Hoefsloot has nothing to disclose. W-J. Koh has nothing to disclose. C. Lange has nothing to disclose. M. Loebinger has nothing to disclose. F.P. Maurer has nothing to disclose. K. Morimoto has nothing to disclose. S. Niemann has nothing to disclose. E. Richter has nothing to disclose. C.Y. Turenne has nothing to disclose. R. Vasireddy has nothing to disclose. S. Vasireddy has nothing to disclose. D. Wagner has nothing to disclose. R.J. Wallace Jr has nothing to disclose. N. Wengenack has nothing to disclose. J. van Ingen has nothing to disclose.

\section{References}

1 Gupta RS, Lo B, Son J. Phylogenomics and comparative genomic studies robustly support division of the genus Mycobacterium into an emended genus Mycobacterium and four novel genera. Front Microbiol 2018; 9: 67.

2 Naushad HS, Lee B, Gupta RS. Conserved signature indels and signature proteins as novel tools for understanding microbial phylogeny and systematics: identification of molecular signatures that are specific for the phytopathogenic genera Dickeya, Pectobacterium and Brenneria. Int J Syst Evol Microbiol 2014; 64: 366-383.

3 Fedrizzi T, Meehan CJ, Grottola A, et al. Genomic characterization of nontuberculous mycobacteria. Sci Rep 2017; 7: 45258.

4 Tortoli E, Fedrizzi T, Meehan CJ, et al. The new phylogeny of the genus Mycobacterium: the old and the news. Infect Genet Evol 2017; 56: 19-25.

5 Oren A, Garrity G. List of new names and new combinations previously effectively, but not validly, published. Int J Syst Evol Microbiol 2018; 68: 1411-1417.

6 International code of nomenclature of prokaryotes. Int J Syst Evol Microbiol 2019; 69: S1-S111.

$7 \quad$ Tindall BJ. Misunderstanding the Bacteriological code. Int J Syst Bacteriol 1999; 49: 1313-1316.

8 Tindall BJ, Kampfer P, Euzeby JP, et al. Valid publication of names of prokaryotes according to the rules of nomenclature: past history and current practice. Int J Syst Evol Microbiol 2006; 56: 2715-2720.

9 Tortoli E. Commentary: Phylogenomic and comparative genomic studies robustly support division of the genus Mycobacterium into an emended genus Mycobacterium and four novel genera. Front Microbiol 2018; 9: 2065.

10 Telenti A. More on "what's in a name ..." - pragmatism in mycobacterial taxonomy. Int J Tuberc Lung Dis 1998; 2: $182-183$.

11 Kwak N, Dalcolmo MP, Daley CL, et al. Mycobacterium abscessus pulmonary disease: individual patient data meta-analysis. Eur Respir J 2019; 54: 1801991.

12 van Ingen J, Aksamit $\mathrm{T}$, Andrejak $\mathrm{C}$, et al. Treatment outcome definitions in nontuberculous mycobacterial pulmonary disease: an NTM-NET consensus statement. Eur Respir J 2018; 51: 1800170.

13 Griffith DE, Eagle G, Thomson R, et al. Amikacin liposome inhalation suspension for treatment-refractory lung disease caused by Mycobacterium avium complex (CONVERT): A prospective, open-label, randomized study. Am J Respir Crit Care Med 2018; 198: 1559-1569.

14 van Ingen J, Turenne CY, Tortoli E, et al. A definition of the Mycobacterium avium complex for taxonomical and clinical purposes, a review. Int J Syst Evol Microbiol 2018; 68: 3666-3677. 